\title{
Biological effects of pyrroloquinoline quinone on liver damage in Bmi-1 knockout mice
}

\author{
YUANQING HUANG $^{1,2}$, NING CHEN $^{1}$ and DENGSHUN MIAO ${ }^{1}$ \\ ${ }^{1}$ State Key Laboratory of Reproductive Medicine, The Research Center for Bone and Stem Cells, \\ Department of Anatomy, Histology and Embryology, Nanjing Medical University, Nanjing, Jiangsu 210029; \\ ${ }^{2}$ Department of Stomatology, Hunan University of Medicine, Huaihua, Hunan 418000, P.R. China
}

Received March 5, 2015; Accepted May 8, 2015

DOI: $10.3892 /$ etm.2015.2532

\begin{abstract}
Pyrroloquinoline quinone (PQQ) has been demonstrated to function as an antioxidant by scavenging free radicals and subsequently protecting the mitochondria from oxidative stress-induced damage. The aim of the present study was to investigate whether PQQ is able to rescue premature senescence in the liver, induced by the deletion of B cell-specific Moloney MLV insertion site-1 (Bmi-1), by inhibiting oxidative stress. In vivo, the mice were allocated into three groups that underwent the following treatment protocols. WT mice received a normal diet, while BKO mice also received a normal diet. An additional group of BKO mice were fed a PQQ-supplemented diet $(\mathrm{BKO}+\mathrm{PQQ} ; 4 \mathrm{mg} \mathrm{PQQ} / \mathrm{kg}$ in the normal diet). The results indicated that $P Q Q$ partially rescued the liver damage induced by the deletion of Bmi-1. PQQ was demonstrated to exhibit these therapeutic effects on liver damage through multiple aspects, including the promotion of proliferation, antiapoptotic effects, the inhibition of senescence, the upregulation of antioxidant ability, the downregulation of cell cycle protein expression, the scavenging of reactive oxygen species and the reduction of DNA damage. The results of these experiments indicated that treatment of BKO mice with a moderate dose of PQQ significantly protected the liver from deleterious effects by inhibiting oxidative stress and participating in DNA damage repair. Therefore, PQQ has great potential as a therapeutic agent against oxidative stress during liver damage.
\end{abstract}

Correspondence to: Professor Dengshun Miao and Professor Ning Chen, State Key Laboratory of Reproductive Medicine, The Research Center for Bone and Stem Cells, Department of Anatomy, Histology and Embryology, Nanjing Medical University, 140 Han Zhong Road, Nanjing, Jiangsu 210029, P.R. China

E-mail: dengshunmiao@126.com

E-mail: njen@njmu.edu.cn

Key words: B cell-specific Moloney MLV insertion site-1, pyrroloquinoline quinone, liver, oxidative stress, antioxidants

\section{Introduction}

The liver is a unique organ that is usually silent under physiological conditions; however, the organ exhibits regenerative properties following damage and/or parenchymal loss (1). In previous years, hepatic disease and the associated morbidity rates have increased year by year, with the condition becoming a major global health care problem (2). A previous study demonstrated that reactive oxygen species (ROS) may be a cause of liver damage, which is characterized by a progression from steatosis to chronic hepatitis, cirrhosis and hepatocellular carcinoma (3). Although studies have attempted to identify efficient liver therapeutics from herbal origins, a number of the potential candidates have not been well characterized and require further investigation (4).

Over 50 years ago, Harman proposed the free radical or oxidative stress theory of aging (5). The author hypothesized that free radicals and/or ROS are produced endogenously from normal cellular metabolic processes. In this theory, an imbalance between ROS and antioxidants can lead to oxidative stress, which subsequently damages various macromolecules. An increasing body of evidence has demonstrated that an increased production of ROS plays an important role in the development of various age-associated diseases (6).

B cell-specific Moloney MLV insertion site-1 (Bmi-1) belongs to the Polycomb group of genes, which are transcriptional repressors that are essential for the maintenance of appropriate gene expression patterns during development (7). The premature deletion of Bmi-1 produces a typical osteoporotic phenotype, and $\mathrm{Bmi}-1^{\%}$ mice have been shown to exhibit a premature aging phenotype of the entire body, including the liver (8). Furthermore, protection against oxidative stress and apoptosis has emerged as an important Bmi-1-downstream pathway, either through the reduction of P53 levels via Bmi-1-mediated repression of the INK4a/Arf locus or via modulation of the oxidative stress response in an INK4a/Arf-independent manner. In Bmi-1\% mice, an increase in ROS coincides with an increase in DNA damage, and subsequently the activation of DNA damage repair pathways. Treatment with $\mathrm{N}$-acetylcysteine or the targeting of CHK2 has been demonstrated to at least partially restore a number of the phenotypes (9). 
Pyrroloquinoline quinone (PQQ) was first identified as a novel cofactor of ethanol and glucose dehydrogenase in methylotrophic bacteria, whereas currently PQQ is considered as an important nutritional growth factor $(10,11)$. PQQ, 4,5-dihydro-4,5-dioxo-1H-pyrrolo [2,3-f] quinoline-2,7,9-tricarboxylic acid, is considered to be a bacterial glucose dehydrogenase redox cofactor that is widely distributed in plants, bacteria, animals, food and numerous biological fluids. PQQ is soluble in water and thermally stable, and can be divided into an oxidized and reduced form $(12,13)$.

Previous studies have demonstrated that PQQ has multiple physiological functions, including the promotion of growth and reproduction (14-16), neural and cardiovascular protection (17-19), and enhancing the learning and memory function (20), immune function, and antitumor effects (21). However, the potential mechanisms underlying these effects remain poorly understood. PQQ has also been reported to function as an antioxidant and pro-oxidant to protect the mitochondria from oxidative stress-induced damages (22-25). A further study defined PQQ as an ROS scavenger in oxidative stress (26).

In recent years, PQQ has become increasingly studied with its role as an antioxidant in the scavenging of superoxide radicals and the protection of the mitochondria from oxidative stress-induced damage. However, the mechanism underlying the effects of PQQ on $\mathrm{Bmi}-1 \%$ mice is yet to be fully elucidated.

In the present study, Bmi-1 knockout (BKO) mice were used to induce a typical liver senescent phenotype. The aim of the study was to investigate whether PQQ was able to restore the premature senescence induced by the deletion of Bmi-1 in the liver through an antioxidative stress pathway.

\section{Materials and methods}

Mice and genotyping. Bmi-1 heterozygote $\left(\mathrm{Bmi}^{-1} 1^{+}\right)$mice ( 2 male and 6 female; average age, 6 weeks; average weight, $24.5 \mathrm{~g}) 129 \mathrm{Ola} / \mathrm{FVB} / \mathrm{N}$ hybrid background) were backcrossed $10-12$ times to the $\mathrm{C} 57 \mathrm{BL} / 6 \mathrm{~J}$ background and mated to generate a Bmi-1 homozygote (Bmi-1/\%; 4 male and 8 female; average age, 7 weeks; average weight, $4.5 \mathrm{~g}$ ), and their wildtype (WT) littermates were genotyped by polymerase chain reaction, as described previously $(8,27)$. One male mouse was paired with 2 female mice to produce newborn Bmi-1 knockout (BKO) and wild type (WT) mice. Male and female mice from the same litter (littermates) were paired with one another. All mice were obtained from the Holland Cancer Institute. Mice were maintained in the Experimental Animal Center of Nanjing Medical University (Nanjing, China). The study was conducted in strict accordance with the guidelines of the Institute for Laboratory Animal Research of Nanjing Medical University, and the experimental protocols were approved by the Committee on the Ethics of Animal Experiments of Nanjing Medical University.

Animal treatment and $P Q Q$ supplementary diet. Purified PQQ was provided by Professor Chunjun Wen from the Academy of Life Sciences of Nanjing Normal University (Nanjing, China). The PQQ-supplemented diet was produced by Beijing KeAo Third Feed Co., Ltd. (Beijing, China). In vivo, the animals were divided into three groups of six mice. The
WT group underwent 3-week weaning littermate WT mice and were fed a normal diet for 4 weeks. Secondly, the BKO group underwent a 3-week weaning littermate BKO mice and were fed a normal diet for 4 weeks. Finally, the BKO + PQQ group underwent 3-week weaning littermate BKO mice and were fed a PQQ-supplemented diet $(4 \mathrm{mg} P Q Q / \mathrm{kg}$ in the normal diet) for 4 weeks (28). After 7 weeks, the three groups of six mice were sacrificed by neck dislocation under ether for further analysis.

Analysis of mice phenotype, percentage survival rate and body weight. In order to investigate the effect of PQQ on the whole body phenotype of the Bmi-1\% mice, the animals were divided into three groups of six mice (WT, BKO and BKO + PQQ). Statistical analysis was conducted on the size, shape, degree of hair smoothness, body weight and percentage survival in the different groups of mice.

Histological examination. Liver tissues were collected and fixed in PLP fixative (2\% paraformaldehyde containing $0.075 \mathrm{~mol} / 1$ lysine and $0.01 \mathrm{~mol} / 1$ sodium periodate) overnight at $4^{\circ} \mathrm{C}$, and processed as described previously (9). All the samples were dehydrated and embedded in paraffin, and sectioned at a 5- $\mu \mathrm{m}$ thickness using an RM2235 rotary microtome (Leica Microsystems, Inc., Buffalo Grove, IL, USA). The sections were stained using standard hematoxylin-eosin (H\&E). Stained tissue sections were assessed for the detection of changes in the magnitude of liver defects using an Olympus GX51 inverted light microscope (Olympus Corporation, Tokyo, Japan).

Immunohistochemical staining. Immunohistochemical staining was conducted for proliferating cell nuclear antigen (PCNA), $\gamma \mathrm{H} 2 \mathrm{AX}, \mathrm{P} 53$ and superoxide dismutase (SOD)2 using the avidin-biotin-peroxidase complex technique with an affinity-purified goat anti-rabbit PCNA antibody (1:400, ab18197; Abcam, Cambridge, UK), an affinity-purified goat anti-mouse $\gamma \mathrm{H} 2 \mathrm{AX}$ antibody (1:200, sc-120804; Santa Cruz Biotechnology, Inc., Santa Cruz, CA, USA), an affinity-purified goat anti-mouse P53 antibody (1:300, \#2524; Cell Signal Technology, Inc., Shanghai, China) and an affinity-purified goat anti-rabbit SOD2 antibody (1:200, ab118340; Abcam), as described previously (9). Briefly, the dewaxed and rehydrated paraffin-embedded sections were incubated with methanol-hydrogen peroxide $(1: 10)$ to block the endogenous peroxidase activity and washed in Tris-buffered saline $(\mathrm{pH}$ 7.6). The slides were subsequently incubated with the primary antibodies overnight at room temperature. Following rinsing with Tris-buffered saline for $15 \mathrm{~min}$, the sections were incubated with a biotinylated secondary antibody (1:100; Sigma-Aldrich, St. Louis, MO, USA). Subsequently, the sections were washed and incubated with the Vectastain Elite $\mathrm{ABC}$ reagent (Vector Laboratories, Inc., Burlington, ON, Canada) for $45 \mathrm{~min}$. After washing, a brown pigmentation was produced using 3,3-diaminobenzidine. Finally, the stained sections were counterstained with H\&E. Images were acquired with a Leica microscope (Leica DM4000B; Leica Microsystems $\mathrm{GmbH}$, Wetzlar, Germany) using Image-Pro Plus, version 5.1 (Media Cybernetics, Inc. Rockville, MD, USA. 
A

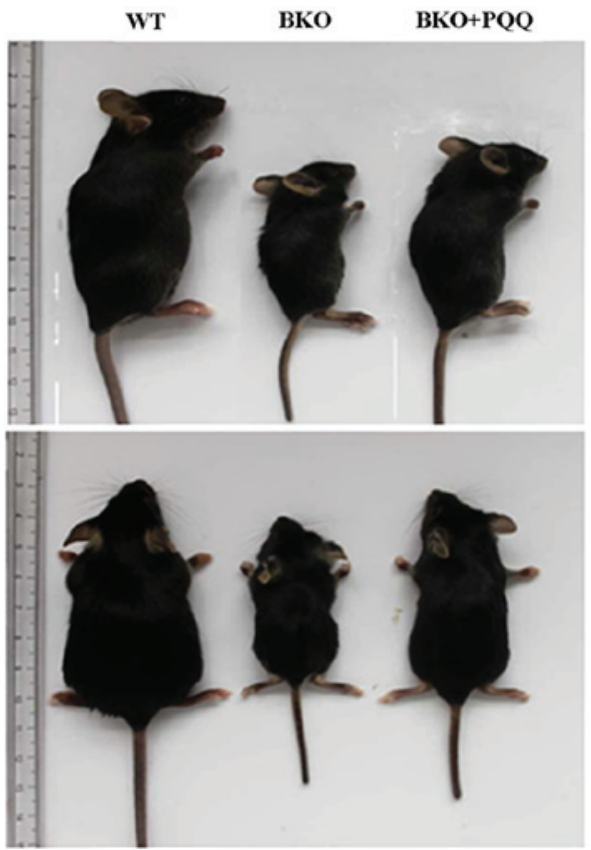

B

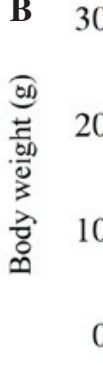

C

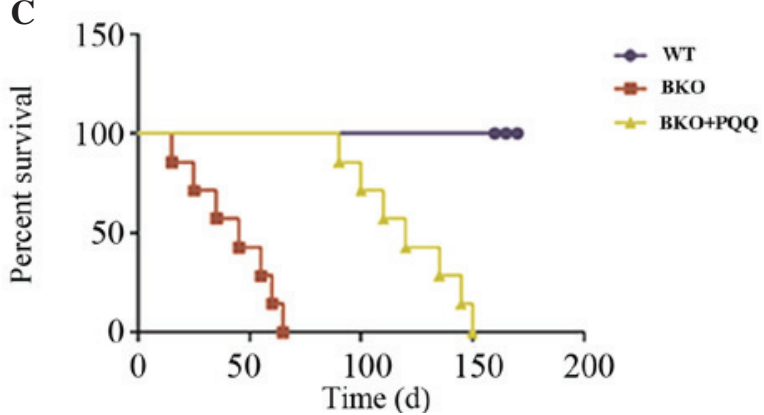

Figure 1. Effects of PQQ on the phenotype, body weight and percentage survival of BKO mice. Effects of a PQQ-supplemented diet on (A) BKO mice size, (B) BKO mice body weight and (C) BKO mice percentage survival. PQQ, pyrroloquinoline quinone; BKO, Bmi-1 ${ }^{-1-} \mathrm{knockout;}$ WT, wildtype; Bmi-1, B cell-specific Moloney MLV insertion site-1.

Western blot analysis. Proteins were extracted from the liver and quantitated using a kit, according to the manufacturer's instructions (Bio-Rad Laboratories, Inc., Mississauga, ON, Canada). Protein samples were fractionated by SDS-PAGE (1\% agarose) and transferred to nitrocellulose membranes (0.45 and $0.22 \mu \mathrm{m}$; Pall Corporation, Port Washington, NY, USA). Western blot analysis was performed as described previously (29), using antibodies against P16 (goat anti-mouse; M-156; 1:400; sc-98520; Santa Cruz Biotechnology, Inc.), P19 (goat anti-mouse; 1:400; sc-271566; Santa Cruz Biotechnology, Inc.), P21 (goat anti-mouse; M19; 1:400; sc-271532; Santa Cruz Biotechnology, Inc.), P27 (goat anti-mouse; 1:400; \#393380; Zymed Laboratories, Santa Cruz, CA, USA), P53 (goat anti-mouse; 1:400, \#2524; Cell Signaling Technology, Inc.), glutathione (GSH; goat anti-mouse; 1:200; sc-292189; Santa Cruz Biotechnology, Inc.), SOD1 and SOD2 (goat anti-rabbit; 1:200; ab20926; Abcam), peroxiredoxin (PRDX) I (goat anti-rabbit; 1:400; ab15571; Abcam), PRDX IV (goat anti-rabbit; $1: 400 ;$ ab59542; Abcam) and $\beta$-actin (goat anti-rabbit; 1:400; sc-130656; Santa Cruz Biotechnology, Inc.). Bands were visualized using enhanced chemiluminescence (GE Healthcare Life Sciences, Shanghai, China), and quantitated using Scion Image Beta 4.02 software (Scion Corporation, Bethesda, MD, USA).

Detection of ROS levels. Liver tissue samples were converted into single cell suspensions containing $5 \times 10^{5}$ cells $/ \mathrm{ml}$. Subsequently, 2',7'-dichlorofluorescein diacetate (DCFH-DA; Sigma-Aldrich) was used for the detection of intracellular ROS. The fluorescence intensity was proportional to the oxidant production (30). DCFH-DA was added to the liver cell suspensions to yield a final concentration of $20 \mu \mathrm{mol} / 1$. Next, the cells were incubated at $37^{\circ} \mathrm{C}$ for $30 \mathrm{~min}$ in the dark, washed twice with $0.01 \mathrm{~mol} / 1$ phosphate-buffered saline, and centrifuged at
$300 \mathrm{x} g$ for $5 \mathrm{~min}$. The ROS levels were measured according to the mean fluorescence intensity of 10,000 cells using a flow cytometer (BD Biosciences, Franklin Lakes, NJ, USA).

Computer-assisted image analysis. Following H\&E staining or histochemical or immunohistochemical staining of the sections from the three groups of six mice each, images of the interested fields were photographed with a SONY digital camera (Sony Corporation, Tokyo, Japan). Images of the micrographs from a single section were digitally recorded using a rectangular template, and recordings were processed and analyzed using Northern Eclipse image analysis software (Empix Imaging, Inc., Mississauga, ON, Canada), as described previously (9).

Statistical analysis. Data are expressed as the mean \pm standard error of the mean. Data were analyzed using SPSS software, version 11.5 (SPSS, Inc., Chicago, IL, USA). Statistical analyses of the numeration data were performed using the $\chi^{2}$ test, while statistical analyses of the measurement data were performed with the Student's $t$-test. $\mathrm{P}<0.05$ was considered to indicate a statistically significant difference.

\section{Results}

Effects of $P Q Q$ on the premature aging phenotype in Bmi-1\% mice. To investigate whether a PQQ-supplemented diet was able to rescue the premature aging phenotype of $\mathrm{Bmi-1 \%}$ mice, statistical analysis of the phenotype, body weight and percentage survival was performed for the different groups of mice. As shown in Fig. 1A-C, when compared with the normal diet WT mice, the BKO mice who were fed a normal diet exhibited a significant premature aging phenotype, body weight loss and a decreased percentage survival rate. By contrast, the 
A

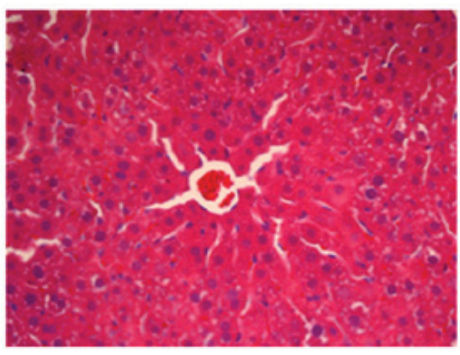

B

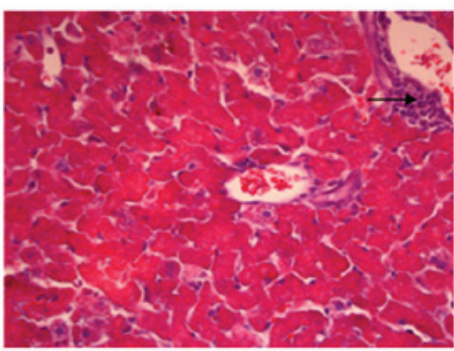

C $\square$ BKO+PQQ

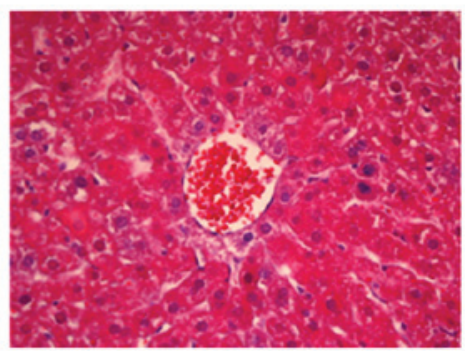

Figure 2. Effects of PQQ on the histological morphology of the liver from BKO mice. (A) Histological morphology of the liver from WT mice (n=6) fed a normal diet. (B) BKO mice $(\mathrm{n}=6)$ fed a normal diet for 4 weeks exhibited a damaged morphology in the liver. (C) BKO mice treated with a PQQ-supplemented diet $(n=6)$ for 4 weeks exhibited reduced damage in the liver compared with the BKO group. Optical microscopy (stain, hematoxylin-eosin; magnification, x400). PQQ, pyrroloquinoline quinone; BKO, Bmi-1/- knockout; WT, wildtype; Bmi-1, B cell-specific Moloney MLV insertion site-1.

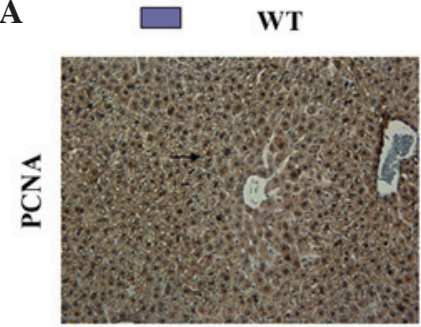

C

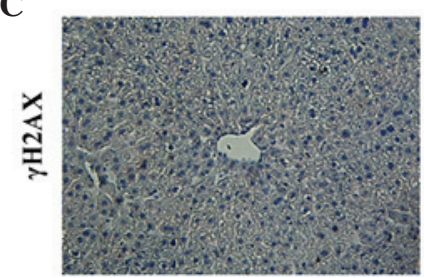

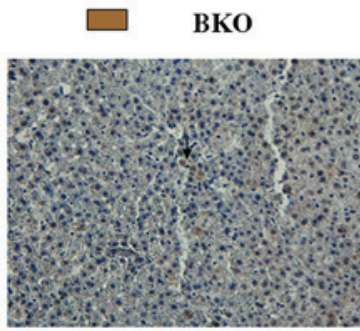

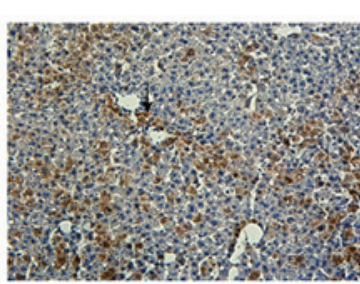

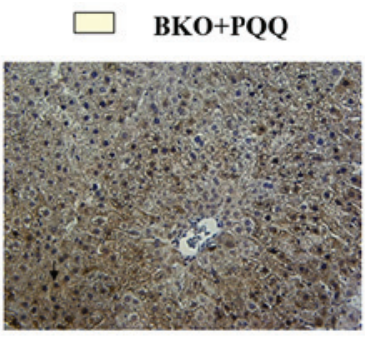

B
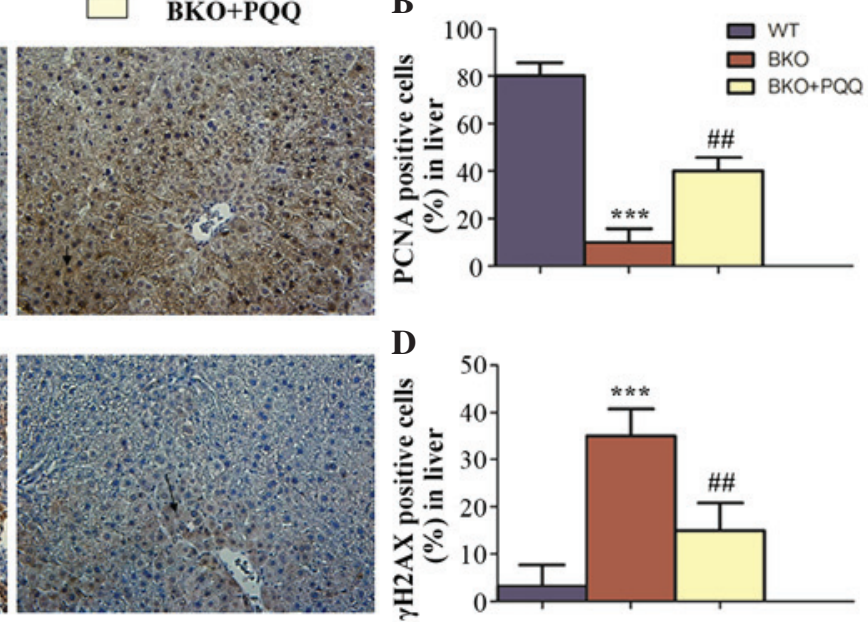

Figure 3. Effects of PQQ on cell proliferation and DNA damage in the liver of BKO mice. (A) Representative liver tissues of the WT, BKO and BKO + PQQ mice stained immunohistochemically for PCNA. (B) Percentage of liver cells stained positive for PCNA in the WT, BKO and BKO + PQQ mice. (C) Representative liver tissues of the WT, BKO and BKO + PQQ mice stained immunohistochemically for $\gamma \mathrm{H} 2 \mathrm{AX}$. (D) Percentage of liver cells stained positive for $\gamma \mathrm{H} 2 \mathrm{AX}$ in the WT, BKO and BKO + PQQ groups. Values are represented as the mean \pm standard error of the mean for the determination of six animals in the same group. ${ }^{* * * *} \mathrm{P}<0.001$, vs. WT mice; ${ }^{\# \#} \mathrm{P}<0.01$, vs. BKO mice. Optical microscopy (magnification, x200). PQQ, pyrroloquinoline quinone; BKO, Bmi-1 ${ }^{-/}$knockout; WT, wildtype; PCNA, proliferating cell nuclear antigen; Bmi-1, B cell-specific Moloney MLV insertion site-1.

BKO mice administered the PQQ-supplemented diet exhibited a partially restored total body size, increased body weight and a prolonged percentage survival when compared with the BKO mice fed the normal diet. These results support the hypothesis that the PQQ-supplemented diet partially restored the premature aging phenotype in the $\mathrm{Bmi}^{-1} \%$ mice when compared with the mice fed a normal diet.

Effects of $P Q Q$ on the histological morphology of the liver in Bmi-1\% mice. Investigations into whether Bmi-1\% mice exhibited an impaired liver histological morphology were performed. As shown in Fig. 2, the histopathology of the liver in the WT mice revealed a normal structure and a regular arrangement of hepatocytes with clearly visible nuclei and a characteristic pattern of hexagonal lobules (Fig. 2A). However, when compared with the WT mice, the BKO mice fed a normal diet exhibited significant pathological alterations, including an irregular arrangement of hepatocytes with invisible nuclei and the presence of increased inflammatory cell infiltration (Fig. 2B). By contrast, the BKO mice administered the PQQ-supplemented diet exhibited good protection against hepatocellular necrosis, with a regular arrangement of hepatocytes (Fig. 2C). These observations indicated that the PQQ-supplemented diet played a protective role in the morphology of the liver damage induced by the deletion of Bmi-1.

Effects of $P Q Q$ on cell proliferation and DNA damage in the liver of Bmi-1\% mice. Since the histological morphology of the hepatocytes was impaired in the Bmi-1\% mice, and this effect was partially restored by the PQQ-supplemented diet, whether the changes in the hepatocytes were caused by the effects of the PQQ-supplemented diet on the rate of cell proliferation was investigated using immunohistochemical staining of PCNA (Fig. 3A). The results revealed that the number of PCNA positively stained cells decreased in the livers of the $\mathrm{BKO}$ mice. However, the PQQ-supplemented diet was shown to significantly enhance the number of PCNA-positive cells in the liver when compared with the $\mathrm{BKO}$ mice fed a normal diet, although the overall levels remained lower than the WT mice (Fig. 3B). A deficiency in the Bmi-1 gene is known to increase the number of DNA double strand breaks via DNA damage repair pathways. To determine whether PQQ reduced the extent of liver DNA 

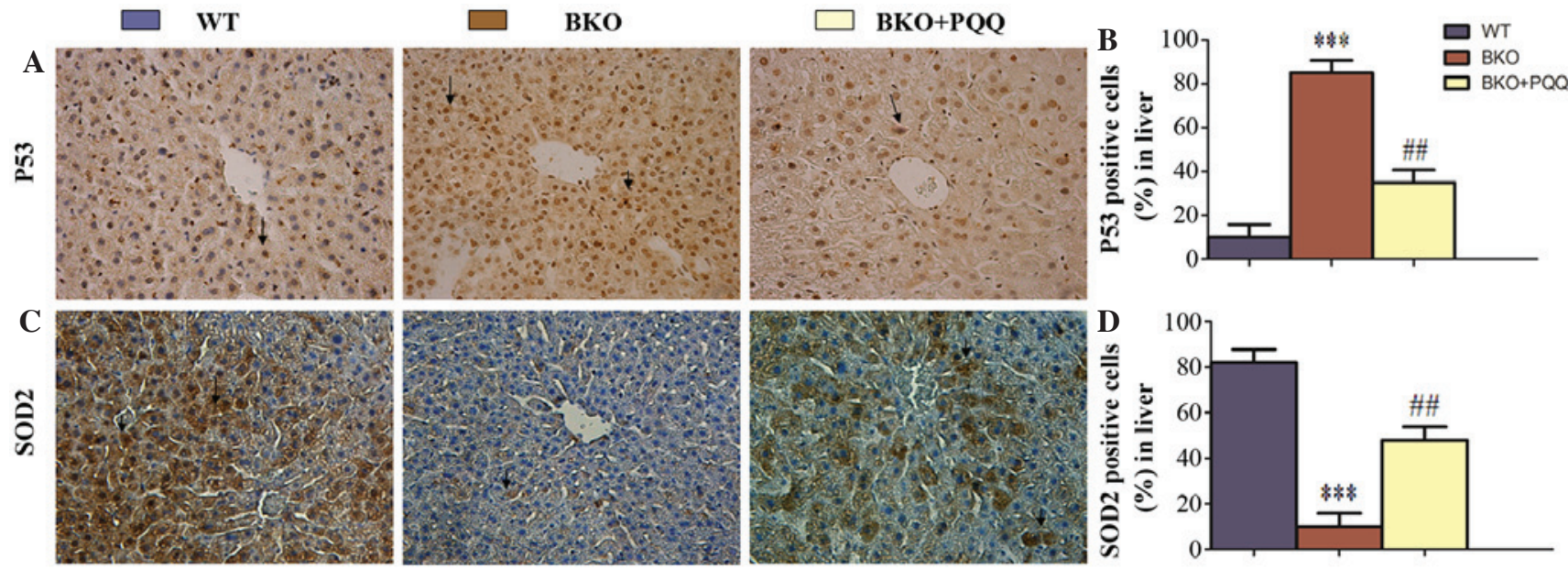

Figure 4. Effects of PQQ on P53 and SOD2 protein expression in the liver of BKO mice. (A) Representative liver tissues of the WT, BKO and BKO + PQQ mice stained immunohistochemically for P53. (B) Percentage of liver cells stained positive for P53 in the WT, BKO and BKO + PQQ mice. (C) Representative liver tissues of the WT, BKO and BKO + PQQ mice stained immunohistochemically for SOD2. (D) Percentage of liver cells stained positive for SOD2 in the $\mathrm{WT}, \mathrm{BKO}$ and $\mathrm{BKO}+\mathrm{PQQ}$ mice. Values are represented as the mean \pm standard error of the mean for the determination of six animals in the same groups. ${ }^{* * * *} \mathrm{P}<0.001$, vs. WT mice; ${ }^{\# \# / P} \mathrm{P}<0.01$, vs. BKO mice. Optical microscopy (magnification, $\mathrm{x} 400$ ). PQQ, pyrroloquinoline quinone; BKO, Bmi-1/- knockout; WT, wildtype; SOD2, superoxide dismutase 2; Bmi-1, B cell-specific Moloney MLV insertion site-1.
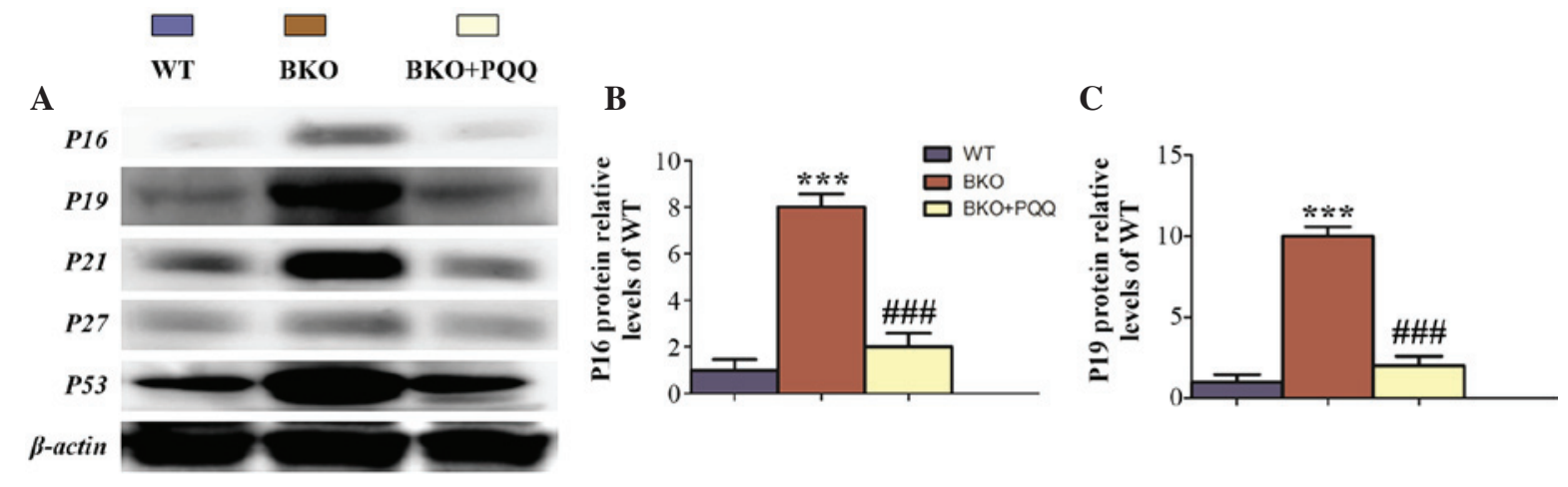

D

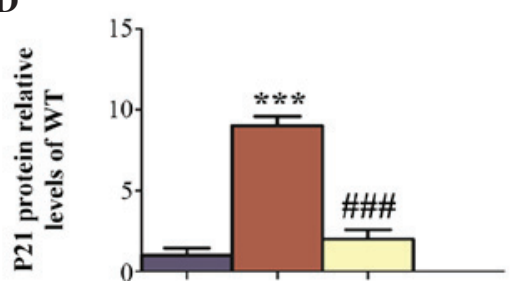

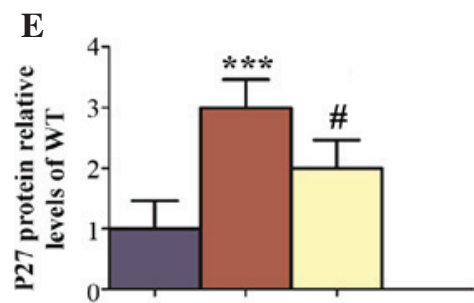

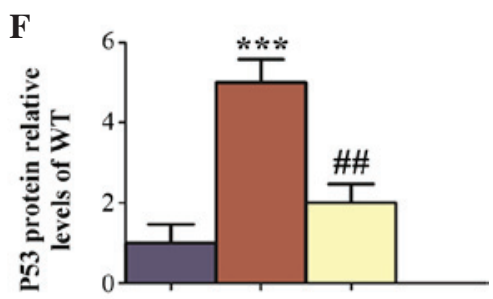

Figure 5. Effects of PQQ on the expression of cell cycle proteins in the liver of BKO mice. (A) Representative western blot showing the protein expression of $\mathrm{P} 16, \mathrm{P} 19, \mathrm{P} 21, \mathrm{P} 27$ and P53 in the liver. $\beta$-actin was used as a loading control for the western blots in the WT, BKO and BKO + PQQ mice. Protein expression levels of (B) P16, (C) P19, (D) P21, (E) P27 and (F) P53, relative to the $\beta$-actin protein levels, were assessed by densitometric analysis and expressed relative to levels of the WT mice. Values are represented as the mean \pm standard error of the mean for the determination of six animals in the same group. ${ }^{* * *} \mathrm{P}<0.001$, vs. WT mice; ${ }^{\#} \mathrm{P}<0.05,{ }^{\# \#} \mathrm{P}<0.01$ and ${ }^{\# \# \#} \mathrm{P}<0.001$, vs. BKO mice. $\mathrm{PQQ}$, pyrroloquinoline quinone; BKO, Bmi-1-1 knockout; WT, wildtype; Bmi-1, B cell-specific Moloney MLV insertion site-1.

damage in the BKO mice, immunohistochemical staining of one of the most commonly used markers for double strand DNA breaks, $\gamma \mathrm{H} 2 \mathrm{AX}$, was performed (Fig. 3C). The results revealed that the BKO mice exhibited significantly higher levels of induced cell DNA damage, as indicated by the protein expression level of $\gamma \mathrm{H} 2 \mathrm{AX}$. However, the increased DNA damage was prevented by administration of the PQQ-supplemented diet (Fig. 3D). Therefore, these results indicated that BKO mice exhibited an increased rate of cell apoptosis by enhancing the double strand DNA damage; however, this effect was ameliorated in the $\mathrm{BKO}$ mice fed the PQQ-supplemented diet.
Effects of PQQ on $P 53$ and SOD2 protein expression in the liver of $\mathrm{Bmi}^{-1 \%}$ mice. Bmi-1 regulates its downstream pathway by reducing the expression levels of cell cycle proteins, or through upregulating the antioxidant ability (9). To determine whether PQQ decreased the protein expression of P53 and increased the expression of SOD2 in the livers of the BKO mice, immunohistochemical staining of P53 and SOD2 proteins was performed (Fig. 4A and C). The results revealed that the number of P53-positive cells increased in the livers of the BKO mice (Fig. 4B). In addition, the PQQ-supplemented diet significantly reduced the number of 
A

Control

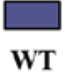

BKO

BKO+PQQ
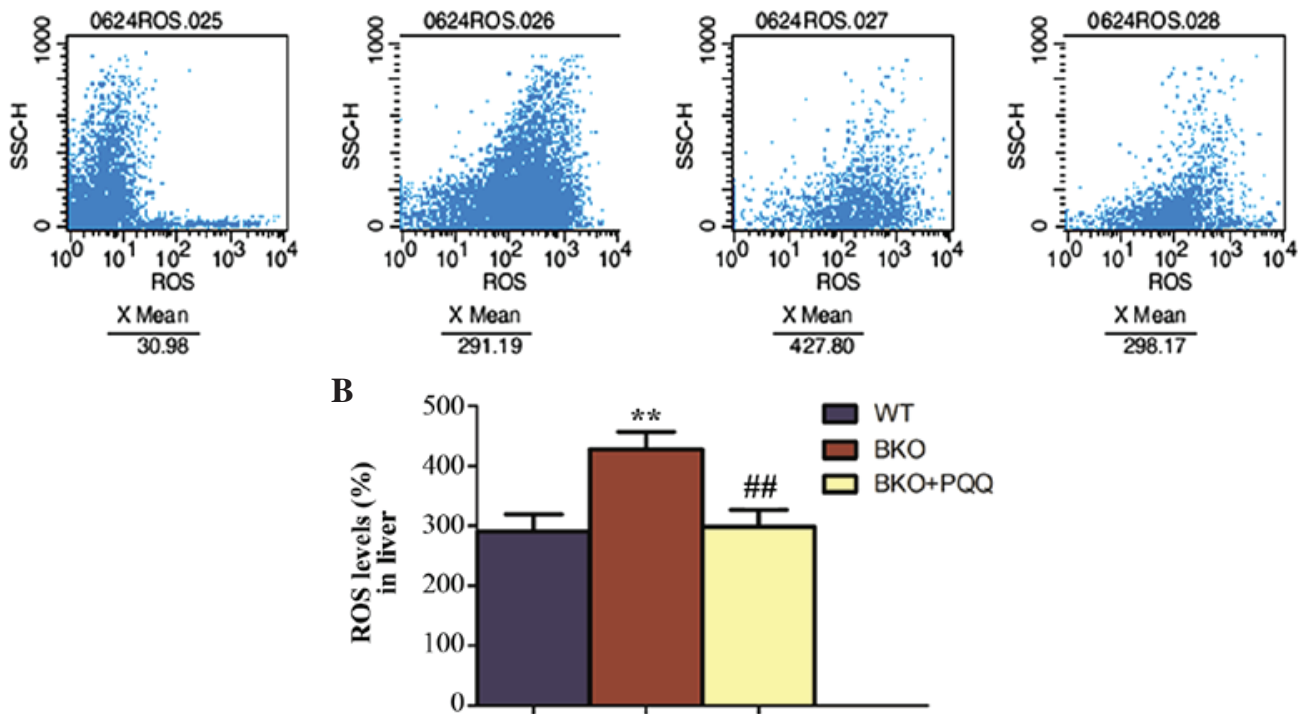

Figure 6. Effects of PQQ on the ROS levels in the liver of BKO mice. (A) Representative flow cytometric analysis showing the ROS levels in the liver of the $\mathrm{WT}, \mathrm{BKO}$ and BKO + PQQ mice. (B) Quantitative determination of the ROS levels in the liver samples. Values are represented as the mean \pm standard error of the mean for the determination of six animals in the same group. ${ }^{* *} \mathrm{P}<0.01$, vs. WT mice; ${ }^{\# \#} \mathrm{P}<0.01$, vs. BKO mice. $\mathrm{PQQ}$, pyrroloquinoline quinone; $\mathrm{BKO}$, Bmi-1 ${ }^{-1}$ knockout; WT, wildtype; ROS, reactive oxygen specie; Bmi-1, B cell-specific Moloney MLV insertion site-1.

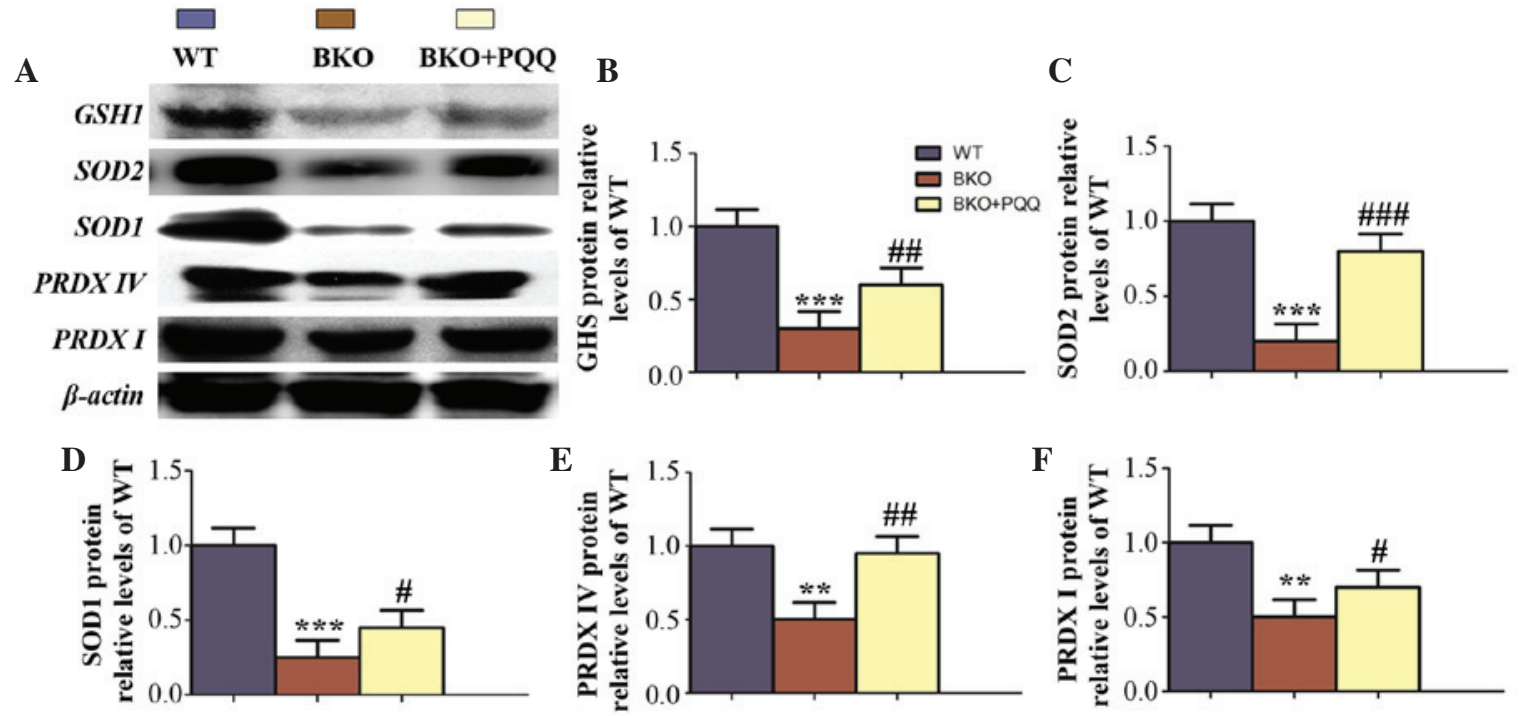

Figure 7. Effects of $\mathrm{PQQ}$ on the protein expression of antioxidants in the liver of $\mathrm{BKO}$ mice. (A) Representative western blot showing the protein expression of GSH, SOD1, SOD2, PRDXI and PRDX IV in the liver. $\beta$-actin was used as a loading control for the western blot in the WT, BKO and BKO + PQQ mice. Protein expression levels of (B) GSH1, (C) SOD2, (D) SOD1, (E) PRDX IV and (F) PRDX, relative to the $\beta$-actin protein levels, were assessed by densitometric analysis and expressed relative to the levels of the WT mice. Values are represented as the mean \pm standard error of the mean for the determination of six animals in the same group. ${ }^{* *} \mathrm{P}<0.01$ and ${ }^{* * *} \mathrm{P}<0.001$, vs. WT mice; ${ }^{\#} \mathrm{P}<0.05,{ }^{\# \#} \mathrm{P}<0.01$ and ${ }^{\# \# \#} \mathrm{P}<0.001$, vs. BKO mice. $\mathrm{PQQ}$, pyrroloquinoline quinone; $\mathrm{BKO}$, Bmi-1/ knockout; WT, wildtype; GSH, glutathione; SOD, superoxide dismutase; PRDX, peroxiredoxin; Bmi-1, B cell-specific Moloney MLV insertion site-1.

P53-positive cells in the BKO + PQQ mice when compared with the BKO mice. However, the number of SOD2-positive cells decreased in the livers of the BKO mice. Notably, the PQQ-supplemented diet was shown to ameliorate the reduction in the expression of the antioxidant proteins in the BKO mice (Fig. 4D). Collectively, these results indicated that a deletion of Bmi-1 induced defects in liver development by inhibiting cell proliferation and downregulating the anti- oxidant ability; however, these effects were partially restored following the administration of the PQQ-supplemented diet in the BKO mice.

Effects of $P Q Q$ on cell cycle protein expression in the liver of Bmi-1\% mice. Cell proliferation is known to be mediated by the expression and activation of tumor-suppressor genes (31); thus, the expression levels of the cell cycle proteins, P16, 
P19, P21, P27 and P53, were examined (Fig. 5A). The results revealed that the BKO mice promoted the expression of the tumor-suppressors, P16, P19, P21, P27 and P53. However, administration of the PQQ-supplemented diet in the BKO mice prevented the increased expression levels of P16, P19, P21, P27 and P53 (Fig. 5B-F).

Effects of PQQ on the ROS levels in the liver of Bmi-1\% mice. Bmi-1\% mice are known to have increased levels of ROS and hydroxyl free radicals that are produced by oxidative stress, which subsequently results in an increase in DNA double strand breaks (32). The present results demonstrated that the PQQ-supplemented diet decreased the number of BKO-induced double strand DNA breaks in the liver. To determine whether PQQ inhibited ROS formation, ROS levels were evaluated by flow cytometry (Fig. 6A). The results revealed that the increased ROS levels in the BKO mice were inhibited by administration of the PQQ-supplemented diet (Fig. 6B).

Effects of $P Q Q$ on the expression levels of antioxidant proteins in the liver of Bmi-1\% mice. To further investigate whether the effects of PQQ were associated with the enhanced expression levels of various antioxidant proteins, western blot analysis was performed to examine the protein expression levels of PRDX I, PRDX IV, SOD1, SOD2 and GSH (Fig. 7A). The results demonstrated that the expression of all the antioxidant proteins were downregulated in the BKO mice when compared with the WT mice, which was consistent with immunohistochemical staining results. Notably, the expression levels of all the antioxidant proteins were increased to a viable level in the BKO + PQQ mice when compared with the BKO mice (Fig. 7B-F). These observations indicated that PQQ promoted an antioxidant effect to protect against BKO-induced oxidative stress.

\section{Discussion}

Bmi-1 is a member of the Polycomb family of transcriptional repressors that mediate gene silencing by regulating chromatin structure, and are essential for the maintenance and self-renewal of hematopoietic and neural stem cells (7-9). Under normal conditions, the Polycomb protein, Bmi-1, simultaneously represses the INK4a/Arf locus, leading to reduced expression levels of P16 $6^{\text {Ink4a }}$ and P19 ${ }^{\text {Arf }}$, as well as modulating mitochondrial function to reduce ROS levels and suppress the activation of the DNA damage response pathway (DDR). Activation of INK4a/Arf and the DDR have been separately associated with tumor suppression and stem cell aging (9). Previous studies have proposed that oxidative stress and the associated damage may represent a common association between different forms of diseases $(33,34)$. Oxidative stress has been implicated in various liver diseases, including viral hepatitis, nonalcoholic fatty liver disease/steatohepatitis, alcoholic liver disease and drug-induced liver injury $(35,36)$.

PQQ, an anionic water soluble compound, was initially proposed to be a cofactor of certain bacterial primary dehydrogenases (37). Subsequently, the compound was attributed with multiple physiological functions, including regulation of the electron transport system and stimulation of the production of nerve growth factor (38). In addition, PQQ has been reported to scavenge ROS and protect cells from oxidative stress-induced damage through improving the activities of free-radical scavenging enzymes and decreasing the levels of free radicals (39). In vitro studies have demonstrated that PQQ protects isolated liver mitochondria from damage following oxidative stress and scavenges superoxide radicals $(40,41)$. Furthermore, an in vivo study investigating ischemia/reperfusion injury in rats demonstrated that PQQ reduces the myocardial infarct size and improves cardiac function (42). In addition, in vivo and in vitro studies have shown that PQQ can protect against several types of oxidative damage, stroke damage and irradiation injury $(19,43)$. However, despite all these beneficial functions of PQQ, to the best of our knowledge, no attempt has been made to evaluate the role of PQQ in the regulation of liver development defects (44). Furthermore, there are a limited number of studies that have investigated whether PQQ can function as a potential ameliorative agent in Bmi-1 deficiency-induced liver dysfunction. In the present study, a liver dysfunctional animal model induced by Bmi-1\% was generated to determine whether PQQ functions as an antioxidant to restore the damage of the liver. The results of the present study, with regard to the pathological, cellular and molecular levels of oxidative stress and antioxidant activity, clearly demonstrate that the administration of a PQQ-supplementary diet, at a dose of $4 \mathrm{mg} \mathrm{PQQ} / \mathrm{kg}$ in the normal diet, to $\mathrm{Bmi}-1 \%$ mice significantly reduces the extent of damage in the liver.

However, there is no effective treatment for the deleterious effects of a number of risk factors on the liver in clinical practice. Therefore, studies have started to focus on substances known as protectors that may inhibit or reduce liver damage. The functions of these protectors are directly targeted to reduce the free radicals formed by oxidative stress. As a powerful antioxidant, PQQ may potentiate to prevent the loss of secretory cells in the liver caused by BKO-induced oxidative stress (45).

In the current study, knowledge on PQQ was further extended, and treatment with a PQQ-supplementary diet was demonstrated to provide robust protection to the liver. As demonstrated by the present results, PQQ is able to rescue the damage to liver morphology, possibly by promoting cell proliferation and inhibiting cell apoptosis and DNA damage. Our further results indicate that PQQ not only inhibits expressions of cell proteins (P16 and P19), cell cycle dependent kinase inhibitors (CDKs, P21 and P27) and apoptotic genes (P53), but also upregulates a variety of antioxidant proteins, such as GSH, SOD1, SOD2, PRDX I and PRDX IV. Simultaneously, the present results also demonstrated that PQQ was able to reduce the ROS levels in an impaired liver. Collectively, the results demonstrated that PQQ, as an antioxidant, is able to inhibit oxidative stress by decreasing the levels of ROS, and rescue cell survival by downregulating the expression of cell cycle proteins, including P16, P19, P21, P27 and P53. Consequently, $\mathrm{PQQ}$ is able to restore the defects of liver development induced in BKO mice.

In conclusion, the results of the present study indicate that treatment of BKO mice with a moderate dose of PQQ significantly protects the liver from deleterious effects by inhibiting oxidative stress and participating in DNA damage repair. Therefore, PQQ has a great potential as a therapeutic agent against oxidative stress during liver damage. 


\section{Acknowledgements}

The study was supported by a grant from the National Natural Science Foundation of China (no. 81230009).

\section{References}

1. Fan $\mathrm{L}, \mathrm{Xu} \mathrm{C}$, Wang $\mathrm{C}$, et al: $\mathrm{Bmil}$ is required for hepatic progenitor cell expansion and liver tumor development. PLoS One 7: e46472, 2012.

2. Park MC, Youn HJ, Chang HK, et al: TOP1 and 2, polysaccharides from Taraxacum officinale, attenuate $\mathrm{CCl}_{4}$-induced hepatic damage through the modulation of NF- $\mathrm{BB}$ and its regulatory mediators. Food Chem Toxicol 48: 1255-1261, 2010.

3. Srivastava A and Shivanandappa T: Hepatoprotective effect of the root extract of Decalepis hamiltonii against carbon tetrachloride-induced oxidative stress in rats. Food Chem 118 411-417, 2010

4. Muriel P, Alba N, Pérez-Alvarez VM, et al: Kupffer cells inhibition prevents hepatic lipid peroxidation and damage induced by carbon tetrachloride. Comp Biochem Physiol C Toxicol Pharmacol 130: 219-226, 2001

5. Harman D: Aging: A theory based on free radical and radiation chemistry. J Gerontol 11: 298-300, 1956.

6. Salmon AB, Richardson A and Pérez VI: Update on the oxidative stress theory of aging: Does oxidative stress play a role in aging or healthy aging? Free Radic Biol Med 48: 642-655, 2010.

7. Park IK, Qian D, Kiel M, et al: Bmi-1 is required for maintenance of adult self-renewing haematopoietic stem cells. Nature 423: 302-305, 2003

8. Zhang HW, Ding J, Jin JL, et al: Defects in mesenchymal stem cell self-renewal and cell fate determination lead to an osteopenic phenotype in Bmi-1 null mice. J Bone Miner Res 25: 640-652, 2010.

9. Liu J, Cao L, Chen J, et al: Bmil regulates mitochondrial function and the DNA damage response pathway. Nature 459: 387-392, 2009

10. Hauge JG: Glucose dehydrogenase of bacterium anitratum: An enzyme with a novel prosthetic group. J Biol Chem 239: 3630-3639, 1964

11. Duine JA: Cofactor diversity in biological oxidations: Implications and applications. Chem Rec 1: 74-83, 2001.

12. Mitchell AE, Jones AD, Mercer RS and Rucker RB Characterization of pyrroloquinoline quinone amino acid derivatives by electrospray ionization mass spectrometry and detection in human milk. Anal Biochem 269: 317-325, 1999.

13. Kumazawa T, Sato K, Seno H, et al: Levels of pyrroloquinoline quinone in various foods. Biochem J 307: 331-333, 1995.

14. Stites TE, Mitchell AE and Rucker RB: Physiological importance of quinoenzymes and the $\mathrm{O}$-quinone family of cofactors J Nutr 130: 719-727, 2000.

15. Steinberg FM, Gershwin ME and Rucker RB: Dietary pyrroloquinoline quinone: Growth and immune response in BALB/c mice. J Nutr 124: 744-753, 1994.

16. Steinberg F, Stites TE, Anderson P, et al: Pyrroloquinoline quinone improves growth and reproductive performance in mice fed chemically defined diets. Exp Biol Med (Maywood) 228 160-166, 2003.

17. Zhang Y, Feustel PJ and Kimelberg HK: Neuroprotection by pyrroloquinoline quinone (PQQ) in reversible middle cerebral artery occlusion in the adult rat. Brain Res 1094: 200-206, 2006.

18. Zhang $\mathrm{Y}$ and Rosenberg PA: The essential nutrient pyrroloquinoline quinone may act as a neuroprotectant by suppressing peroxynitrite formation. Eur J Neurosci 16: 1015-1024, 2002.

19. Zhu BQ, Simonis U, Cecchini G, et al: Comparison of pyrroloquinoline quinone and/or metoprolol on myocardial infarct size and mitochondrial damage in a rat model of ischemia/reperfusion injury. J Cardiovasc Pharmacol Ther 11: 119-128, 2006.

20. Ohwada K, Takeda H, Yamazaki M, et al: Pyrroloquinoline quinone (PQQ) prevents cognitive deficit caused by oxidative stress in rats. J Clin Biochem Nutr 42: 29-34, 2008.

21. Shankar BS, Pandey R, Amin P, et al: Role of glutathione in augmenting the anticancer activity of pyrroloquinoline quinone (PQQ). Redox Rep 15: 146-154, 2010
22. Ouchi A, Nakano M, Nagaoka S and Mukai K: Kinetic study of the antioxidant activity of pyrroloquinolinequinol (PQQH (2), a reduced form of pyrroloquinoline quinone) in micellar solution. J Agric Food Chem 57: 450-456, 2009.

23. Stites T, Storms D, Bauerly K, et al: Pyrroloquinoline quinone modulates mitochondrial quantity and function in mice. J Nutr 136: 390-396, 2006.

24. Ishii T, Akagawa M, Naito Y, et al: Pro-oxidant action of pyrroloquinoline quinone: Characterization of protein oxidative modifications. Biosci Biotechnol Biochem 74: 663-666, 2010.

25. Tao R, Karliner JS, Simonis U, et al: Pyrroloquinoline quinone preserves mitochondrial function and prevents oxidative injury in adult rat cardiac myocytes. Biochem Biophys Res Commun 363: 257-262, 2007

26. Misra HS, Khairnar NP,Barik A, et al: Pyrroloquinoline-quinone: A reactive oxygen species scavenger in bacteria. FEBS Lett 578: 26-30, 2004.

27. Cao G, Gu M, Zhu M, et al: Bmi-1 absence causes premature brain degeneration. PLoS One 7: e32015, 2012.

28. Bauerly K, Harris C, Chowanadisai W, et al: Altering pyrroloquinoline quinone nutritional status modulates mitochondrial, lipid, and energy metabolism in rats. Plos One 6: e21779, 2011.

29. Xue Y, Karaplis AC, Hendy GN, et al: Genetic models show that parathyroid hormone and 1,25-dihydroxyvitamin D3 play distinct and synergistic roles in postnatal mineral ion homeostasis and skeletal development. Hum Mol Genet 14: 1515-1528, 2005.

30. Zamzami N, Marchetti P, Castedo M, et al: Sequential reduction of mitochondrial transmembrane potential and generation of reactive oxygen species in early programmed cell death. J Exp Med 182: 367-377, 1995.

31. Bae I, Fan S, Bhatia K, Kohn KW, Fornace AJ Jr and O'Connor PM: Relationships between G1 Arrest and Stability of the $\mathrm{p} 53$ and $\mathrm{p} 21 \mathrm{CiP} 1 /$ Waf1 Proteins following gamma-irradiation of human lymphoma cells. Cancer Res 55: 2387-2393, 1995.

32. Di Pietro C, Piro S, Tabbi G, et al: Cellular and molecular effects of protons: Apoptosis induction and potential implications for cancer therapy. Apoptosis 11: 57-66, 2006.

33. Sano R and Reed JC: ER stress-induced cell death mechanisms. Biochim Biophys Acta 1833: 3460-3470, 2013.

34. Tang W, Jiang YF, Ponnusamy M and Diallo M: Role of Nrf2 in chronic liver disease. World J Gastroenterol 20: 13079-13087, 2014.

35. Weltman MD, Farrell GC, Hall P, et al: Hepatic cytochrome P450 $2 \mathrm{E} 1$ is increased in patients with nonalcoholic steatohepatitis. Hepatology 27: 128-133, 1998.

36. Okuda M, Li K, Beard MR, et al: Mitochondrial injury, oxidative stress and antioxidant gene expression are induced by hepatitis $\mathrm{C}$ virus core protein. Gastroenterology 122: 366-375, 2002.

37. Salisbury SA, Forrest HS, Cruse WB and Kennard O: A novel coenzyme from bacterial primary alcohol dehydrogenases. Nature 280: 843-844, 1979.

38. Rucker R, Chowanadisai W and Nakano M: Potential physiological importance of pyrroloquinoline quinone. Altern Med Rev 14: 268-277, 2009.

39. Killgore J, Smidt C, Duich L, et al: Nutritional importance of pyrroloquinoline quinone. Science 245: 850-852, 1989.

40. Bishop A, Paz MA, Gallop PM, et al: Methoxatin PQQ in guinea-pig neutrophils. Free Radic Biol Med 17: 311-320, 1994.

41. He K, Nukada H, Urakami T and Murphy MP: Antioxidant and pro-oxidant properties of pyrroloquinoline quinone (PQQ): Implications for its function in biological systems. Biochem Pharmacol 65: 67-74, 2003

42. Zhu B, Zhou H, Teerlink JR, et al: Pyrroloquinoline quinone (PQQ) decreases myocardial infarct size and improves cardiac function in rat models of ischemia and ischemia/reperfusion. Cardiovasc Drugs Ther 18: 421-431, 2004.

43. Aizenman E, Hartnett KA, Zhong C, et al: Interaction of the putative essential nutrient pyrroloquinoline quinone with the N-methyl-D-aspartate receptor redox modulatory site. J Neurosci 12: 2362-2369, 1992.

44. Kumar N, Kar A and Panda S: Pyrroloquinoline quinone ameliorates l-thyroxine-induced hyperthyroidism and associated problems in rats. Cell Biochem Funct 32: 538-546, 2014

45. Jin J, Lv X, Chen L, Zhang W, Li J, Wang Q, Wang R, Lu X and Miao D: Bmi-1 plays a critical role in protection from renal tubulointerstitial injury by maintaining redox balance. Aging Cell 13: 797-809, 2014. 